\title{
A successful potential technique to reduce prolonged hospital stay of patients associated with the use of continuous drainage follow- ing surgical evacuation of a chronic subdural haematoma (SDH)
}

\author{
H.K.A. Ruwanchinthani ${ }^{1}$, H. Kularathne ${ }^{2}$ \\ 1. Medical Officer, Neuro-Surgical Unit, National Hospital of Sri Lanka, Colombo. \\ 2. Consultant Neurosurgeon; Neuro-Surgical Unit, National Hospital of Sri Lanka, Colombo.
}

\begin{abstract}
Key words: Subdural Hematoma; SDH; Drainage
\section{Introduction}

A Subdural Hematoma (SDH) is a common neurosurgical condition that often requires surgical intervention. It is a type of intracranial hemorrhage that occurs beneath the dura (essentially, a collection of blood over the surface of the brain), and may be associated with concurrent brain injuries. A SDH usually forms after rupture of a bridging vein.
\end{abstract}

It is further categorized into three stages based on the duration. An acute SDH is a rapid collection of blood clot found below the inner layer of the dura but external to the brain and arachnoid membrane. The next two stages, subacute and chronic, may develop with untreated acute SDH. In general, the subacute phase begins 3-7 days after the acute injury. The chronic phase begins about 2-3 weeks after the acute injury. The incidence of chronic subdural hematoma is about 5 per 100000 per year in the general population and higher in those aged 70 years or older [1].

SDH is considered to be associated with high mortality and morbidity rates, even with the best medical and neurosurgical care [2]. Surgical evacuation via craniotomy is often considered in patients with an acute SDH, thicker than 5 $\mathrm{mm}$ (as measured with axial computed tomography scanning), and who have any neurologic signs such as lethargy, change in mental status or focal neurologic deficits. Bullock et al reported that "an acute SDH with a thickness greater than $10 \mathrm{~mm}$, or a midline shift greater than $5 \mathrm{~mm}$ on computed tomography scan should be surgically evacuated, regardless of the patient's GCS score [3].

Various surgical methods have been recommended for the treatment of SDH and are under research. One time burrhole drainage and continuous closed drainage are methods

Correspondence: H.K.A. Ruwanchinthani, Neuro-

Surgical Unit, National Hospital of Sri Lanka, Colombo.

E-mail: amaliruwanchinthani@gmail.com that have been compared recently. The recurrence rate after an initial drainage procedure range from 5\% to 30\% [1], and treatment strategies for reducing recurrence rates are the focus of current research. We report practice of a new technique in the therapy of SDH, which will reduce the long hospital stay and promote early recovery.

\section{Case report}

A 69 year old male patient was admitted to the NeuroSurgical Unit at the National Hospital of Sri Lanka (NHSL) with a history of an accidental fall to the ground, where he had a blunt head impact to the occipital region of the head, with transitional loss of consciousness. At the time of the incident a magnetic resonance scan (MRI) was performed in the private medical sector and was found to be normal except for a scalp haematoma. Later, he developed headache, slurred speech, memory impairment and weakness of the right side of the body which progressively worsened over seven days of duration.

He was reviewed in the private medical sector and an immediate non-contrast computed tomographic (NCCT) scan of the brain (figure 1.1) was performed which revealed a left sided acute-on-chronic subdural haematoma (SDH) with a midline shift of more than $5 \mathrm{~mm}$. On admission to the NHSL his Glasgow Coma Scale (GCS) was 14/15 (E4 V4 M6) and he had a right sided weakness of the body with grade 4 muscle power. His other clinical parameters were within the normal range. He had no other comorbid conditions.

He was immediately prepared for the trephine craniotomy and surgical evacuation of the SDH was performed under general anaesthesia. After evacuating the SDH, the cavity was filled with saline, and then closed with a continuous subdural drain, which was applied under atmospheric pressure. The drain volume was measured and documented. The total 24 hour drainage was $435 \mathrm{cc}$ on the first post operative day, which reduced to 220cc in 48 hours. Initially, the drain content was blood stained, which became clear by the second day. 


\section{Chronic subdural haematoma}

\section{Technique}

Before terminating the closed continuous subdural drain system, our usual protocol has been to wait until the drain is 10cc or less. Usually, this has taken between 7 and 10 days. In our patient we removed the drain 48 hours after operation. Informed consent was obtained before the procedure, which was done in the ward under aseptic conditions, with the patient lying supine. The drain was removed by applying negative pressure through gradual suction using a 5cc syringe. This was an air tight procedure - a volume of 12 cc of clear fluid followed by 4cc of altered blood was removed and there was no active bleeding noted at this point. After removal of the drain sutures were applied to the scalp.

\section{Investigations}

Two NCCT scans were performed, one before and after suction. The patient was observed for twenty four hours after removing the drain. He made a complete recovery on the third post operative day and was discharged on oral medication.

With this procedure of suction of fluid with negative pressure before removing the drain we were able to facilitate early recovery as it helped brain expansion mainly in an elderly patient with cortical atrophy which is demonstrated in the NCCTs below. In the post operative pre-aspiration NCCT (figure 2.1), a left sided white opacity suggestive of recent bleeding, probably following surgery, and an air filled space is demonstrated. By contrast, in the post operative post aspiration NCCT (figure 2.2), expansion of the brain is noted after drainage of air and blood.
On discharge from the hospital, the patient's symptoms were relieved, the GCS was 15/15 and his right side weakness had improved. At regular follow up in the neurosurgical clinic for six months, he did not have post operative infection, recurrence of SDH or deterioration of consciousness.

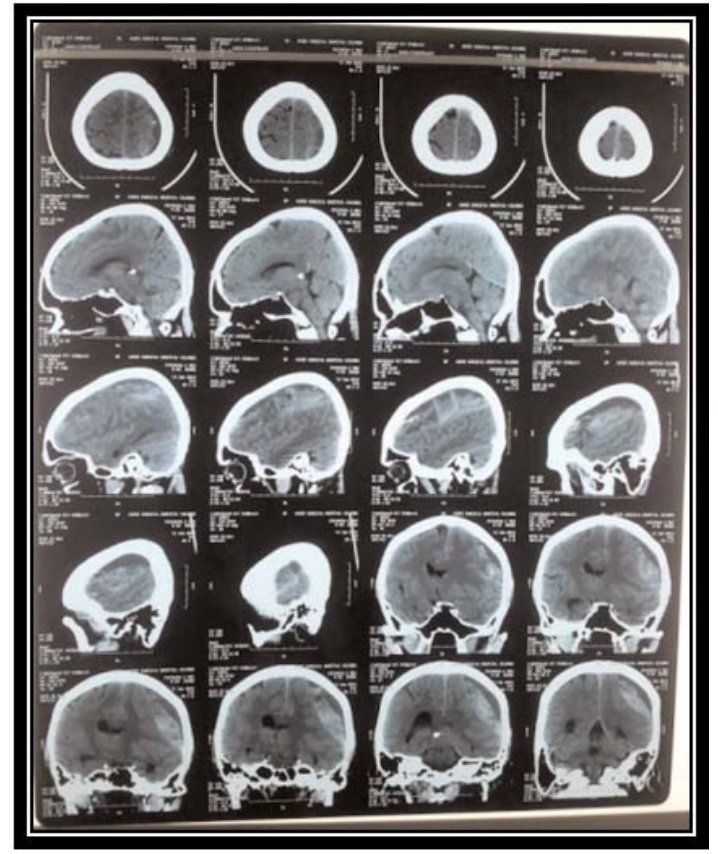

Figure 1. Pre-operative NCCT

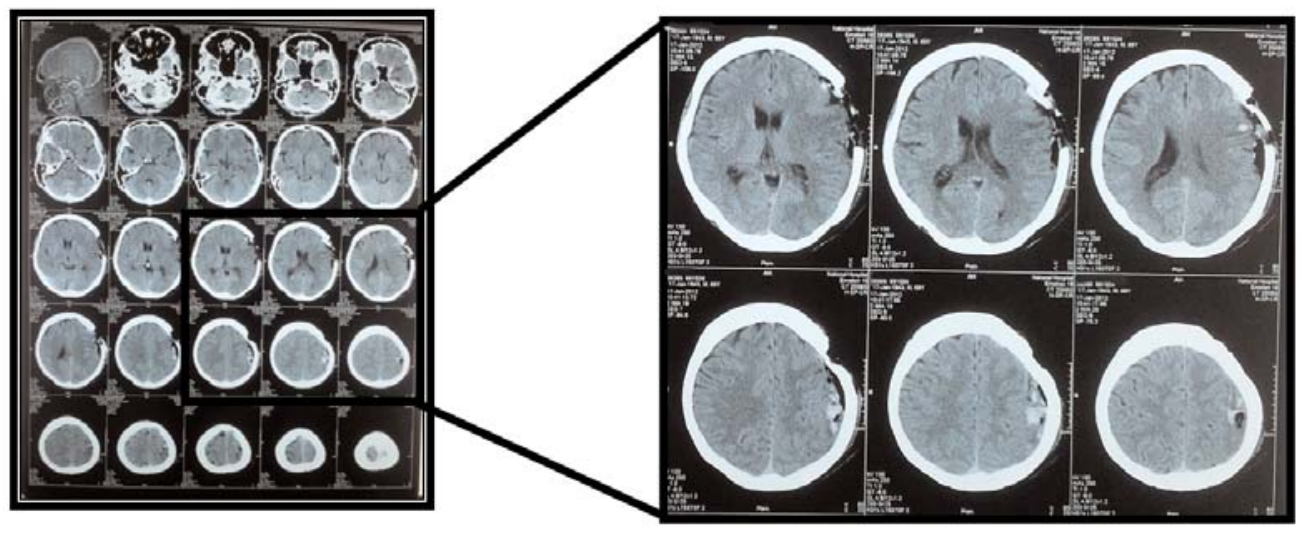

Figure 2. NCCT done on post-operative Day 2 - Pre aspiration 


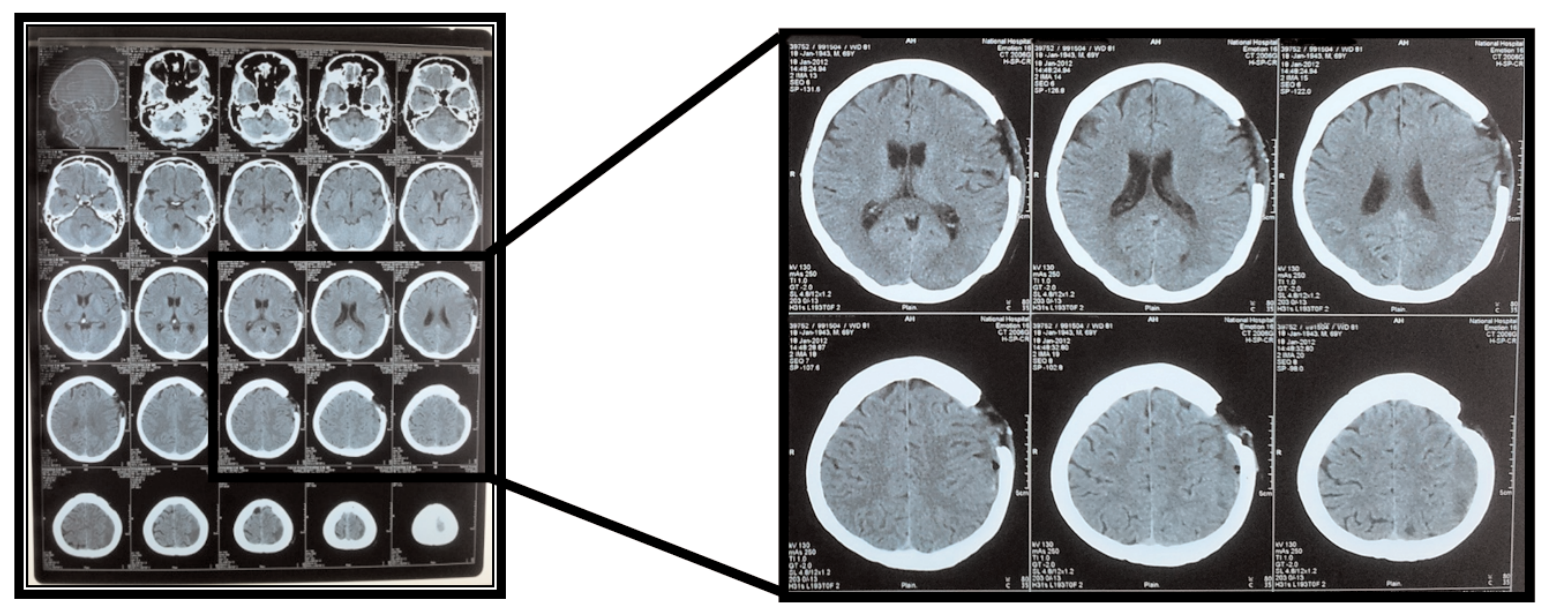

Figure 3. NCCT done on post-operative Day 3 - Post aspiration

\section{Discussion}

A variety of surgical techniques have been described for chronic SDH. Trephination is a common method that is being used. In this procedure, a liquefied chronic SDH is commonly treated with drainage through a single or two burr holes. The burr holes are placed so that conversion to a craniotomy is possible, if needed. A closed drainage system is sometimes left in the subdural space postoperatively. In a case series by Mori and Maeda, $89.4 \%$ of patients with chronic subdural hematoma (SDH) who were treated with a closed drainage system had a good recovery and $2.2 \%$ worsened. Old age, preexisting cerebral infarction, and subdural air after surgery correlated with poor brain expansion [4]. This highlights that removal of subdural air with negative pressure application helps in brain expansion - an observation which is confirmed in our new technique.

A study on controversies in SDH: continuous drainage versus one time drainage done in the department of neurosurgery in Turkey has concluded that burr-hole and continuous drainage (CD) therapy for $\mathrm{SDH}$ is superior to the burr-hole and one time drainage (OTD) method due to shorter time of hospitalization and the reduced rate of recurrence [5]. The post operative hospitalization period was 7.9 days in the CD group and 17 days in the OTD group. Recurrence developed in two cases (6.8\%) in the
CD group and six cases (28.5\%) in the OTD group.

Similarly, in a study done in Cambridge, Santarious-et-al, found that use of an irrigating drain with a burr hole was associated with lower recurrence rate, better neurological status and lower mortality at six months compared with no drain [6, 7].

In the aforementioned studies only one time drainage or continuous drainage was tested, where as in the new method suggested, a combination of closed continuous drainage for forty eight hours was followed by removal of the drain applying negative pressure. As found in the study done in Turkey, the usual hospital stay of 7.9 days with continuous drainage, this new technique has the potential to reduce hospital stay to 3 days.

On the other hand, a more recent comparative study done in New Delhi, India, did not find a statistically significant difference in recurrence rates and concluded that continuous drainage of an SDH did not offer any advantage over simple burr-hole drainage [1].

This emphasizes that continuous drainage only prolongs hospital stay, and this new method of removing the drain after forty eight with negative pressure may be more economical. It has a better prognosis and, most importantly, reduces duration of hospital stay, which is cost effective for the patient as well as the healthcare provider. 


\section{References}

1. Ahmed S, Agrawal D, Kale SS, Mahapatra AK, A comparative study of treatment of chronic subdural hematoma - burr hole drainage versus continuous closed drainage. The Indian Journal of Neurotrauma. 2011; 8: 17-23

2. van den Doel EM. Balzac's 'Pierette'. An early description of chronic subdural hematoma. Arch Neurol. Dec 1986; 43(12):1291-2.

3. Bullock MR, Chesnut R, Ghajar J, Gordon D, Hartl $\mathrm{R}$, Newell DW, et al. Surgical management of acute subdural hematomas [Guideline]. Neurosurgery. 2006; 58 (Suppl):S16-24;

4. Mori K, Maeda M. Surgical treatment of chronic subdural hematoma in 500 consecutive cases: Clinical characteristics, surgical outcome, complications, and recurrence rate. Neurol Med Chir 2001;41(8):371-81.
5. Kiymaz N, Yilmaz N, Mumcu C. Controversies in chronic subdural hematoma: continuous drainage versus one-time drainage. Med Sci Monit. 2007; 13(5): 240-243.

6. Kolias AG, Sinha R, Park H, Santarius T, Hutchinson PJ. Surgical management of chronic subdural haematomas: in need of better evidence. Acta Neurochir (Wien). 2013; 155: (1): 183 -184.

7. Kolias AG, Scotton WJ, Belli A, King AT, Brennan $\mathrm{PM}$, Bulters DO, Eljamel MS, Wilson $\mathrm{MH}$, Papadopoulos MC, Mendelow AD, Menon DK, Hutchinson PJ; UK Neurosurgical Research Network; RESCUE-ASDH collaborative group.

Surgical management of acute subdural haematomas: current practice patterns in the United Kingdom and the Republic of Ireland. $\mathrm{Br} \mathrm{J}$ Neurosurg. 2013 Jun;27(3):330-3. 